\title{
Anti-inflammatory effects of the Portulaca oleracea hydroalcholic extract on human peripheral blood mononuclear cells
}

\author{
Esmaeil Allahmoradi ${ }^{1,2^{\dagger}}$, Saeid Taghiloo ${ }^{1,2^{\dagger}}$, Versa Omrani-Nava ${ }^{1,2}$, Saeideh Sadat Shobeiri ${ }^{1,2}$, Mohsen Tehrani ${ }^{2}$, \\ Mohammad Ali Ebrahimzadeh ${ }^{1,3}$, Hossein Asgarian-Omran*2,4
}

Received: 23 Apr 2017

Published: 3 Sep 2018

\section{Abstract}

Background: Portulaca oleracea, known as Purslane, is an annual growing herb with wide distribution around the world and traditionally used to manage several diseases. Different therapeutic properties as an anti-fever agent as well as anti-inflammatory and analgesic effects have been attributed to $P$. oleracea. The aim of this study was to investigate the effects of $P$. oleracea aerial extract on production of pro- and anti-inflammatory cytokines by human peripheral blood mononuclear cells (PBMCs).

Methods: Aerial parts of $P$. oleracea (stems and leaves) were collected and extracted by percolation using methanol. The optimal and non-cytotoxic dose of hydro-alcoholic extract for cell culture analysis was determined by MTT assay. To assess the antiinflammatory effects of $P$. oleracea, PBMCs obtained from 12 normal volunteers were cultured in RPMI complete medium and cotreated with $E$. coli lipopolysaccharide (LPS) and $P$. oleracea hydro-alcoholic extract. Following 18-hour incubation, culture supernatants were harvested for measurement of secreted TNF- $\alpha$, IL- 6 and IL-10 by ELISA. Statistical analyses were performed using the SPSS v.20, and data analyzed by Kolmogorov-Smirnov, Mann-Whitney U, Kruskal-Wallis and post Hoc tests. P-values $<0.05$ were considered significant.

Results: The optimal non-cytotoxic concentration of $P$. oleracea aerial extract was defined as $100 \mu \mathrm{g} / \mathrm{ml}$ based on MTT viability assay. $P$. oleracea hydro-alcoholic extract significantly decreased the concentration of both pro-inflammatory cytokines TNF- $\alpha$ and IL-6 in LPS-stimulated PBMCs ( $<<0.001$ and $p<0.001$, respectively). However, the concentration of IL-10 as an anti-inflammatory cytokine, did not show any statistically significant change $(\mathrm{p}=0.390)$.

Conclusion: Our findings highlighted the potential anti-inflammatory properties of $P$. oleracea in herbal medicine. Future analysis on different constituents of total extract may confirm its therapeutic effects as a promising anti-inflammatory compound.

Keywords: Portulaca oleracea, Anti-inflammatory agent, Tumor necrosis factor-alpha, Interleukin-6, Interleukin-10

Copyright@ Iran University of Medical Sciences

Cite this article as: Allahmoradi E, Taghiloo S, Omrani-Nava V, Shobeiri SS, Tehrani M, Ebrahimzadeh MA, Asgarian-Omran H. Antiinflammatory effects of the Portulaca oleracea hydroalcholic extract on human peripheral blood mononuclear cells. Med J Islam Repub Iran. 2018 (3 Sep);32:80. https://doi.org/10.14196/mjiri.32.80

\section{Introduction}

Inflammation is a biological protective response to traumatic stimuli, pathogens and damaged cells (1). During inflammation, regular physiological and immunological processes coordinate by soluble signaling molecules of the immune system. Subsequently, the corresponding cells

Corresponding author: Dr Hossein Asgarian-Omran, asgarianhossein@yahoo.com

1. Student Research Committee, Mazandaran University of Medical Sciences, Sari, Iran.

2. Department of Immunology, School of Medicine, Mazandaran University of Medical Sciences, Sari, Iran.

3. Pharmaceutical Sciences Research Center, School of Pharmacy, Mazandaran University of Medical Sciences, Sari, Iran.

4. Immunogenetics Research Center, School of Medicine, Mazandaran University of Medical Sciences, Sari, Iran.

† Esmaeil Allahmoradi and Saeid Taghiloo have contributed equally to this study. are transferred to inflamed sites to resolve the atypical state and finally cause the healing process (2). Following removal of stimuli agents, inflammatory response must be restricted to prevent any uncontrolled damage and further possible autoimmune disorders such as rheumatoid arthri-

$\uparrow$ What is "already known" in this topic:

Different therapeutic properties as an anti-fever agent as well as anti-inflammatory and analgesic effects have been attributed to $P$. oleracea. The anti-inflammatory effects of $P$. oleracea were also investigated in several studies but no data are available regarding its direct anti-inflammatory effects on human peripheral blood mononuclear cells (PBMCs).

$\rightarrow$ What this article adds:

Our preliminary experiments demonstrated for the first time that $P$. oleracea exhibit the immunomodulatory effects by suppression of pro-inflammatory cytokines such as TNF- $\alpha$ and IL6 on LPS-stimulated human PBMCs. 
tis, Crohn's disease, psoriatic arthritis, systemic lupus erythematosus and multiple sclerosis $(3,4)$. Therapeutic interventions would then be necessary to prevent the progression of inflammation without any side effects on the normal host immune response $(1,5)$. Recently, application of traditional herbs as potent anti-inflammatory agents has been dramatically increased as treatment or prevention strategies $(4,6)$. Nevertheless, numerous traditional herbs do not have any scientific documentations and approval to be administered for human which it calls more investigations in this area.

Portulaca oleracea (P. oleracea, Family Portulacaceae), also known as Purslane, is an annual growing herb listed in the World Health Organization as one of the most used therapeutic plants $(7,8)$. P. oleracea is an edible herb and widely distributed plant and commonly used as a traditional medicine in the Middle East, Mediterranean, United States, Central European and Asian countries (9, 10). They possess green color, slightly acidic with branched and cylindrical shape, stems up to $15-30 \mathrm{~cm}$ height and $3 \mathrm{~mm}$ in diameter $(10,11)$. According to the photochemical analysis, Purslane extract is a rich source of fatty acids particularly omega-3, glutathione, glutamic acid, aspartic acid and vitamins including $\mathrm{A}, \mathrm{B}, \mathrm{C}$ and etc. $(2,9,12)$. Several pharmacological properties of $P$. oleracea like anti-oxidant and anti-microbial $(7,13)$, skeletal muscle relaxant (14), wound-healing (10), as well as a traditional medicine for diuretic, febrifuge, sepsis, spasms, headache, scurvy, asthma and fever have been addressed in previous studies $(7,8,11,15,16)$. In addition, other pharmacological actions such as neuroprotectivity, bronchodilator, hepatoprotectivity, anti-fatigue and antihypertensive activities have also been attributed to $P$. oleracea $(13,17,18)$. The anti-oxidant and antiproliferative effects of $P$. oleracea have been demonstrated on several cancer cell lines $(15,19)$. The antiinflammatory effects of Oleracimine, a novel carbon skeleton alkaloid from $P$. oleracea, has been studied on LPSinduced RAW 264.7 cell line by inhibiting the production of pro-inflammatory cytokines such as IL-6, TNF- $\alpha$ as well as nitric oxide (NO) and prostaglandin-E2 (20). Similar anti-inflammatory properties were also reported for Oleracone which constitute other skeleton alkaloid constituent of $P$. oleracea (21). Taking these considerations into accounts, although the anti-inflammatory effects of $P$. oleracea were documented in several studies $(22,23)$, there is no data regarding its direct anti-inflammatory effects on human peripheral blood mononuclear cells (PBMCs).

In the present study, the potential anti-inflammatory properties of $P$. oleracea on human PBMCs were explored to more clarify its pharmacological mechanisms and possible effects on host immune responses.

\section{Methods}

\section{Preparation of plant extract}

P. oleracea was collected from Qeidar Mountains (Khodabandeh, Zanjan province, Iran) in May 2015. The sample was authenticated by Dr. Bahman Eslami (Plant systematic specialist, Islamic Azad University of
Qhaemshahr, Iran) and the voucher specimen was deposited (No. HS181) in the Sari School of Pharmacy Herbarium. Aerial parts (leaves and stems) of the fresh plant were dried at room temperature and coarsely ground before extraction. Dried materials were powdered using blender and then extracted at room temperature by maceration using methanol for 24 hours. The extract was then separated from the sample residue by filtration through filter paper. This procedure was repeated three times. The resultant extracts were concentrated under vacuum (Rotary Evaporator) until a crude solid extract obtained and then freeze-dried for complete solvent removal (16.5\%). The dried extract was dissolved in phosphate buffer saline (PBS 0.15M pH: 7.4) and stored in $4^{\circ} \mathrm{C}$ as plant extract stock.

\section{Study subjects}

A total of 12 healthy normal volunteers including 6 males and 6 females with the mean age of $39.2 \pm 16.65$ years were incorporated in this study. All subjects were clinically evaluated to exclude any underlying diseases or consumption of anti-inflammatory or immunosuppressive drugs. Heparinized peripheral blood samples were taken from all subjects and subsequently used for PBMC isolation. The current study was confirmed by Ethical Committee of Mazandaran University of Medical Sciences and written informed consents were obtained from all participants.

\section{Separation of peripheral blood mononuclear cells}

PBMCs were isolated from fresh peripheral blood of all subjects using Ficoll-Histopaque (Biosera, Nuaille, France). Heparinized whole blood was diluted in equivalent volume of RPMI-1640 culture medium (Biosera, Nuaille, France), then carefully layered over a ficoll density gradient medium and centrifuged at $400 \mathrm{~g}$ for $20 \mathrm{~min}$. Isolated PBMCs were washed twice with RPMI-1640 and applied for further experiments. The viability of isolated cells was $98 \%$ as assessed by trypan blue staining.

Determination of the optimum and non-cytotoxic dose of P. oleracea hydro-alcoholic extract for cell culture analysis

To determine the non-cytotoxic dose of $P$. oleracea hydro-alcoholic extract, $2 \times 10^{5}$ PBMCs were seeded at flatbottomed 96-well microplates (SPL, South Korea) in duplicate and treated with different concentrations of extract from 12.5 to $500 \mu \mathrm{g} / \mathrm{ml}$ for 24 hours at $37{ }^{\circ} \mathrm{C}$ in $5 \% \mathrm{CO} 2$. Phytohemagglutinin (PHA) was also used as a positive control of cell proliferation at $2 \mu \mathrm{g} / \mathrm{ml}$. Cells viability was measured by MTT colorimetric assay (Sigma-Aldrich, Missouri, USA). Following incubation, MTT reagent was added to each well at final concentration of $0.5 \mathrm{mg} / \mathrm{ml}$ and cells were incubated at $37{ }^{\circ} \mathrm{C}$ for 4 hours until purple precipitate was visible. Culture plates were centrifuged at $300 \mathrm{~g}$ for $10 \mathrm{~min}$ and supernatants were removed. Finally, MTT crystals were dissolved in $150 \mu \mathrm{l}$ of DMSO (Biosera, Nuaille, France) and shook at room temperature for 20 min. Optical densities were recorded at $570 \mathrm{~nm}$ with multi scan plate reader (Synergy H1 BioTek, Winooski, USA). 


\section{Cell culture and stimulation}

To assess the effects of $P$. oleracea hydroalcholic extract on cytokine production from PBMCs, $2 \times 10^{5}$ cells were cultured in $200 \mu \mathrm{l}$ RPMI-1640 medium in flatbottomed 96-well microplates supplemented with penicillin $(100 \mathrm{IU} / \mathrm{ml})$, streptomycin $(100 \mu \mathrm{g} / \mathrm{ml})$ and $10 \%$ heat inactivated fetal bovine serum (Biosera, Nuaille, France). The cells were treated with $E$. coli Lipopolysaccharide (LPS-100 $\mathrm{pg} / \mathrm{ml}$ ), as a potent stimulator of proinflammatory cytokines production, together with 100 $\mu \mathrm{g} / \mathrm{ml}$ of $P$. oleracea extract. Non-stimulated and LPSstimulated PBMCs were applied as control wells to compare the results. The effects of P. oleracea extract on production of pro- and anti-inflammatory cytokines by PBMCs were studied in two conditions. At initial experiments, PBMCs were cultured and treated with P. oleracea extract without LPS in a non-inflammatory condition and then, the potential anti-inflammatory properties of $P$. oleracea extract evaluated on LPS-stimulated PBMCs. All cultures were run in duplicate and incubated at $37^{\circ} \mathrm{C}$ in $5 \% \mathrm{CO} 2$ for 18 hours. Culture supernatants were harvested and stored at $-20^{\circ} \mathrm{C}$ for cytokines measurement.

\section{Cytokines measurement by ELISA}

Following an 18-hour incubation, culture supernatants were collected and the concentrations of TNF- $\alpha$ and IL- 6 as pro-inflammatory cytokines and IL-10 as an antiinflammatory cytokine were measured by ELISA kits using the manufacturer's protocol (Sanquin, Amsterdam, Netherlands). All samples were measured in duplicate. All ELISA kits were based on a sandwich assay following by detection with streptavidin-HRP conjugate with the sensitivity of $2 \mathrm{pg} / \mathrm{ml}$. The absorbance of all plates was recorded at $450 \mathrm{~nm}$ as well as $630 \mathrm{~nm}$ as reference wavelength with microplate spectrophotometer.

\section{Statistical Analysis}

Statistical analyses were performed using the SPSS statistical package version 20 (SPSS, Chicago, USA). Data are represented as mean \pm standard error of the mean (SEM). Normality distribution of the obtained data was determined by Kolmogorov-Smirnov test, and the MannWhitney U test used to calculate the mean difference between two groups. For comparison between three groups, the Kruskal-Wallis and post Hoc tests were applied. Pvalues $<0.05$ were considered to be significant.

\section{Results}

To determine the optimum and non-cytotoxic dose of $P$. oleracea hydro-alcoholic extract for cell culture analysis, PBMCs were treated with different concentrations of crude extract and cells viability was determined by MTT assay. PBMCs were proliferated in response to PHA as a polyclonal activator and mitogen for peripheral blood lymphocytes. Based on OD values obtained from MTT assay, a slight proliferation of PBMCs was observed following treatment with lower concentrations of Portulaca oleracea extract compared to control wells (Fig. 1). But, this proliferative response was not statistically significant

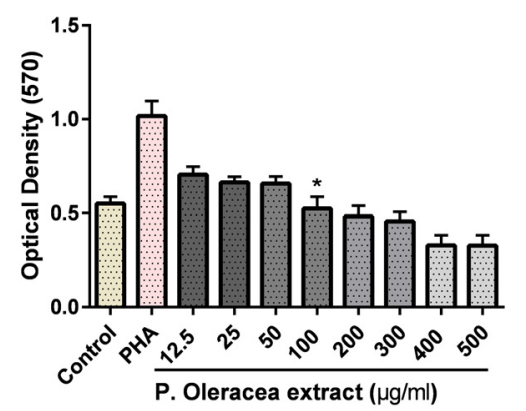

Fig. 1. The non-cytotoxic dose of P. oleracea hydro-alcoholic extract for cell culture analysis determined by MTT assay. Human PBMCs were isolated and treated with serial concentrations of P. oleracea crude extract for 24 hours and cell viability was determined by MTT assay. PHA was used as a positive control and polyclonal mitogen.

*Concentration of $100 \mu \mathrm{g} / \mathrm{ml}$ was selected as non-cytotoxic dose for all experiments.

for any of the extract doses. P. oleracea extract did not show any cytotoxic effects up to $100 \mu \mathrm{g} / \mathrm{ml}$ based on cells viability result. Thus, this concentration was selected for further experimental procedures (Fig. 1).

As represented in Fig. 2, the levels of all measured proand anti-inflammatory cytokines including TNF- $\alpha$, IL- 6 and IL-10 were statistically similar in PBMCs stimulated with $P$. oleracea extract compared to untreated cells ( $\mathrm{p}=$ $0.112, p=0.083$ and $p=0.862$, respectively). In the next experiments, the potential anti-inflammatory properties of $P$. oleracea extract were evaluated on LPS-stimulated PBMCs in the inflammatory condition. To address this issue, PBMCs from all volunteers were co-treated with $P$. oleracea and LPS as well as with LPS alone. While the production of TNF- $\alpha(p=0.009)$ and IL-6 $(p=0.003)$ was significantly elevated by PBMCs in response to LPS, the concentration of IL-10 did not show any difference after stimulation with LPS (Fig. 3A-3C). Interestingly, $P$. oleracea dramatically suppressed the production of TNF- $\alpha$ $(p=0.042)$ and IL-6 $(p=0.010)$ in LPS-stimulated PBMCs (Fig. 3A and 3B) which indicate its potential antiinflammatory effects. However, LPS-induced PBMCs were similarly produced IL-10 in the presence or absence of extract (Fig. 3C).

\section{Discussion}

Inflammation is associated with increasing many inflammatory mediators such as cytokines, chemokines and nitric oxide produced during inflammatory response to stimuli $(1,2)$. TNF- $\alpha$, IL-1 $\beta$ and IL- 6 are the major proinflammatory cytokines playing essential roles in the inflammatory response. Besides their protective effector functions in acute inflammatory conditions, extra production of these cytokines in autoimmune and inflammatory diseases could be harmful and pathologic $(2,24)$. Recent studies have been reported that immunomodulatory agents like medicinal plants provide an alternative therapy for a variety of inflammation-mediated disorders like rheumatoid arthritis and atherosclerosis (6). Portulaca oleracea is a well-defined plant in traditional medicine and has been addressed in previous studies for its various therapeutic 
A.

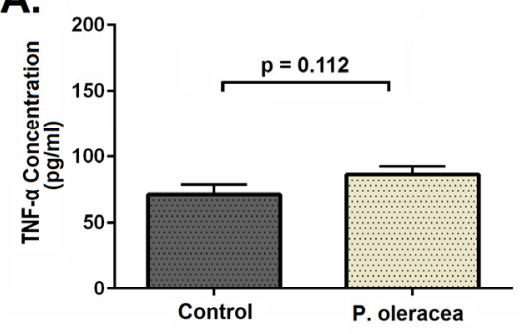

B.

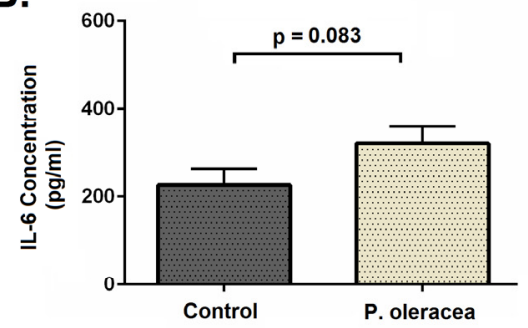

C.

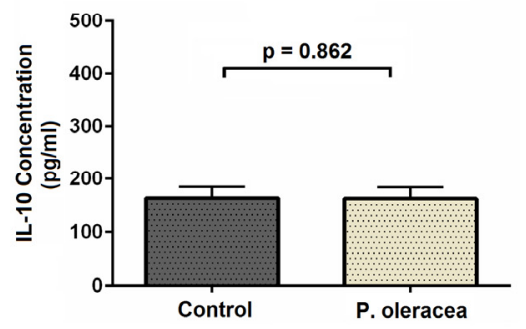

Fig. 2. P. oleracea effects on production of pro- and anti-inflammatory cytokines by human PBMCs measured by ELISA. Isolated PBMCs from normal volunteers $(\mathrm{n}=12)$ were treated with $100 \mu \mathrm{g} / \mathrm{ml}$ of P. oleracea extract for 18 hours and the production of TNF- $\alpha$ (A), IL-6 (B) and IL-10 (C) were measured in culture supernatants by ELISA. The results are represented as mean $\pm \mathrm{SEM}$. P-values $<0.05$ were considered significant.
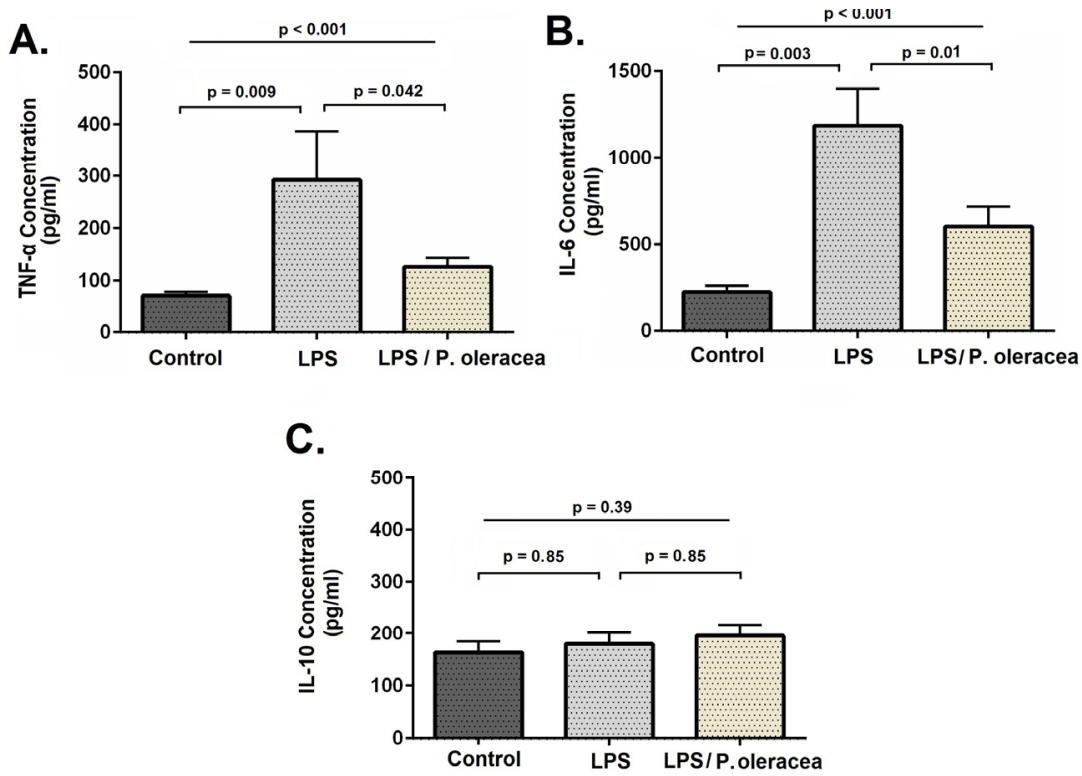

Fig. 3. P. oleracea extract has anti-inflammatory effects on LPS-stimulated human PBMCs. Human PBMCs were isolated from normal volunteers $(\mathrm{n}=12)$ and co-stimulated with LPS $(100 \mathrm{pg} / \mathrm{ml})$ and P. oleracea extract $(100 \mu \mathrm{g} / \mathrm{ml})$ for 18 hours as well as LPS alone. Culture supernatants were collected and used for measurement of TNF- $\alpha$ (A), IL-6 (B) and IL-10 (C) by ELISA. The results are represented as mean \pm SEM. P-values $<0.05$ were considered significant.

applications (9). Our results for the first time demonstrated the anti-inflammatory effects of $P$. oleracea on human PBMCs by down-regulation of TNF- $\alpha$ and IL- 6 production.

TNF- $\alpha$ is mainly produced by mononuclear phagocytes in response to bacterial, viral and parasitic infections. However, aberrant and over-production of TNF- $\alpha$ may stimulate the secretion of other inflammatory mediators resulting DNA and cell damage $(3,25)$. Previous studies have reported that blocking of TNF- $\alpha$ signaling pathway decrease the amounts of inflammatory mediators and amelioration of inflammatory diseases like Crohn's disease, rheumatoid arthritis, ankylosing spondylitis and inflammatory bowel disease (IBD) (25). Inhibition of TNF- $\alpha$ can be achieved by various approaches such as monoclonal antibodies and fusion proteins which some of them are now clinically approved for treatment of TNF- $\alpha$ mediated complications. Together with TNF- $\alpha$, IL- 6 has also critical roles in immune responses to infections during inflammation and over-production of IL-6 has been observed in some pathological conditions including rheumatoid arthritis, osteoporosis, IBD, Crohn's disease and psoriasis $(1,3$, 26). Our initial experiments demonstrated that optimal and selected dose of $P$. oleracea extract $(100 \mu \mathrm{g} / \mathrm{ml})$ was not 
cytotoxic for PBMCs based on the viability assay and also had no effects on production of pro- and antiinflammatory cytokines by PBMCs. To create an inflammatory environment, PBMCs were stimulated with LPS, as an strong inducer of cytokines production by inflammatory cells such as monocytes and macrophages $(3,27)$. Over-production of TNF- $\alpha$ and IL- 6 by LPS-induced PBMCs was remarkably suppressed by co-treatment with $P$. oleracea extract in our experiments. Our results are in line with the previous studies demonstrating the antiinflammatory effects of novel alkaloid constituents from $P$. oleracea named as Oleracone and Oleracimine by inhibition of IL- 6 and TNF- $\alpha$ in LPS-induced RAW264.7 macrophage cell line (21). In addition, it was shown that polysaccharide extract from $P$. oleracea could significantly decrease TNF- $\alpha$ and IL-6 levels in diabetic rats (28) as well as exhibit effective protection for Ulcerative colitis in mice by down-regulation of IL-6, IL- $1 \beta$ and TNF- $\alpha$ (29). In other study by Ramadan et al., it has been reported that pretreatment with the aqueous extract of $P$. oleracea induced significant reduction of TNF- $\alpha$ and IL-6 in diabetic rats (30). The present results demonstrate that the antiinflammatory properties of $P$. oleracea on human PBMCs could be more extended and confirm the previous findings with different approaches.

IL-10 is mainly known as an anti-inflammatory cytokine which suppress the production of pro-inflammatory cytokines including TNF- $\alpha$, IL- 6 and regulate immune responses (31). Blocking of IL-10 signaling pathway during acute infections increases the pathology and susceptibility to inflammatory and autoimmune disorders. Conversely, stimulation of IL-10 production by antiinflammatory agents has therapeutic effects (32-34). In the present study, the concentration of IL-10 did not change in the supernatants of PBMCs treated with P. oleracea or LPS alone and also co-treated with LPS and $P$. oleracea compared to untreated control cells. Since, there are no published reports regarding the effects of $P$. oleracea on IL-10 production, our results in regard to lack of effect of $P$. oleracea extract on IL-10 production call on more studies with different approaches. Taken together, our data indicates that in non-inflammatory conditions, $P$. oleracea has not any cytotoxic and pro-inflammatory effects. But together with the initiation of inflammation like by LPS, $P$. oleracea restrict the inflammatory conditions by suppression of the pro-inflammatory cytokines production. Since, prolonged clinical use of conventional antiinflammatory drugs such as non-steroidal antiinflammatory drugs (NSAIDs) and slow acting antiinflammatory drugs (SAARDs) have many unwanted and harmful side effect (6), our preliminary study showed that $P$. oleracea could be applied as an alternative candidate for routine anti-inflammatory conditions with minimal unwanted side effects. However, future works are required to determine the effective constituents of the crude extract of $P$. oleracea, and its potential therapeutic effects on inflammatory diseases. The current study was financially limited to investigate the immunomodulatory therapeutic effects of $P$. oleracea on animal model for inflammatory diseases and also on PBMCs from patients with inflamma- tory diseases.

\section{Conclusion}

Our promising preliminary experiments have demonstrated the immunomodulatory effects of $P$. oleracea aerial extract by suppression of pro-inflammatory cytokines on LPS-stimulated human PBMCs. These findings could suggest $P$. oleracea potential application as a useful therapeutic candidate for the treatment of inflammation mediated diseases.

\section{Acknowledgements}

This study was financially supported by a grant (No: 184-7) from the Student Research Committee of Mazandaran University of Medical Sciences.

\section{Conflict of Interests}

The authors declare that they have no competing interests.

\section{References}

1.Fry DE. Sepsis, systemic inflammatory response, and multiple organ dysfunction: the mystery continues. Am Surg. 2012;78(1):1-8.

2. Choi W-S, Jeong J-W, Kim SO, Kim G-Y, Kim B-W, Kim CM, et al. Anti-inflammatory potential of peat moss extracts in lipopolysaccharide-stimulated RAW 264.7 macrophages. Int J Mol Med. 2014;34(4):1101-9.

3. Gayathri B, Manjula N, Vinaykumar K, Lakshmi B, Balakrishnan A. Pure compound from Boswellia serrata extract exhibits antiinflammatory property in human PBMCs and mouse macrophages through inhibition of TNF $\alpha$, IL-1 $\beta$, NO and MAP kinases. Int J Immunopharmacol. 2007;7(4):473-82.

4. Smith TJ. Insulin-like growth factor-I regulation of immune function a potential therapeutic target in autoimmune diseases? Pharmacol Rev . 2010;62(2):199-236.

5. Scheller J, Chalaris A, Schmidt-Arras D, Rose-John S. The pro-and anti-inflammatory properties of the cytokine interleukin-6. BBA-Mol Cell Res. 2011;1813(5):878-88.

6. Alamgir M, Uddin SJ. Recent advances on the ethnomedicinal plants as immunomodulatory agents. Ethnomed. 2010;37:227-44.

7.Lim Y, Quah E. Antioxidant properties of different cultivars of Portulaca oleracea. Food chem. 2007;103(3):734-40.

8. Karimi G, Khoei A, Omidi A, Kalantari M, Babaei J, Taghiabadi E, et al. Protective effect of aqueous and ethanolic extracts of Portulaca oleracea against cisplatin induced nephrotoxicity. Iran J Basic Med Sci. 2010;13(2):31-5.

9. Dweck AC. Purslane (Portulaca oleracea)-the global panacea. Pers Care Mag. 2001;2(4):7-15.

10. Zhou YX, Xin HL, Rahman K, Wang SJ, Peng C, Zhang H. Portulaca oleracea L.: a review of phytochemistry and pharmacological effects. Biomed Res Int. 2015;2015.

11. Oliveira I, Valentão P, Lopes R, Andrade PB, Bento A, Pereira JA. Phytochemical characterization and radical scavenging activity of Portulaca oleraceae L. leaves and stems. Microchem J. 2009;92(2):129-34.

12. Yazici I, Türkan I, Sekmen AH, Demiral T. Salinity tolerance of purslane (Portulaca oleracea L.) is achieved by enhanced antioxidative system, lower level of lipid peroxidation and proline accumulation. Environ Exper Bot. 2007;61(1):49-57.

13. Mousavi SM, Bagheri G, Saeidi S. Antibacterial activities of the hydroalcoholic extract of portulaca oleracea leaves and seeds in sistan region, Southeastern Iran. Int J Infect. 2015;2(2).

14. Masoodi MH, Ahmad B, Mir SR, Zargar BA, Tabasum N. Portulaca oleracea L. a review. J Pharm Res. 2011;4:3044-8.

15. Payudara S, Dan Nasofarinks K, Tan G, Wong K, PEArLE-WoNG $\mathrm{GQ}$, Yeo $\mathrm{S}$, et al. In vitro Cytotoxic and antiproliferative effects of Portulaca oleracea methanol extract on breast, cervical, colon and nasopharyngeal cancerous cell lines. Sains Malays. 2013;42:927-35.

16. Boroushaki MT, Boskabady MH, Malek F. Antitussive effect of 
Portulaca oleracea L. in guinea pigs. Iran J Pharm Res. 2010:187-90.

17. Masoodi MH, Ahmad B, Mir SR, Zargar BA, Tabasum N. Portulaca oleracea L. a review. J Pharmacy Res. 2011;4(9):3044-8.

18. Changizi-Ashtiyani S, Zarei A, Taheri S, Rasekh F, Ramazani M. The effects of Portulaca oleracea alcoholic extract on induced hypercholesteroleomia in rats. Zahedan J Res Med Sci. Zahedan J Res Med Sci. 2013;15(6):34-9.

19. Guo G, Yue L, Fan S, Jing S, Yan LJ. Antioxidant and Antiproliferative Activities of Purslane Seed Oil. J hypertens : open access. 2016 Jun;5(2).

20. Li CY, Meng YH, Ying ZM, Xu N, Hao D, Gao MZ, et al. Three Novel Alkaloids from Portulaca oleracea L. and Their Antiinflammatory Effects. J agric food chem. 2016 Jul 27;64(29):583744. PubMed PMID: 27396870.

21. Meng Y, Ying Z, Xiang Z, Hao D, Zhang W, Zheng Y, et al. The anti-inflammation and pharmacokinetics of a novel alkaloid from Portulaca oleracea L. J Pharm Pharmacol. 2016.

22. Sanja S, Sheth N, Patel N, Patel D, Patel B. Characterization and evaluation of antioxidant activity of Portulaca oleracea. Int J Pharm Pharm Sci. 2009;1(1):74-84.

23. Shen H, Tang G, Zeng G, Yang Y, Cai X, Li D, et al. Purification and characterization of an antitumor polysaccharide from Portulaca oleracea L. Carbohydr Polym. 2013;93(2):395-400.

24. Xian YF, Li YC, Ip SP, Lin ZX, Lai XP, Su ZR. Anti-inflammatory effect of patchouli alcohol isolated from Pogostemonis Herba in LPSstimulated RAW264. 7 macrophages. Exp Ther Med. 2011;2(3):54550.

25. Bradley JR. TNF-mediated inflammatory disease. The Journal of pathology. 2008 Jan;214(2):149-60. 26. Neurath MF, Finotto S. IL-6 signaling in autoimmunity, chronic inflammation and inflammationassociated cancer. Cytokine growth F R. 2011;22(2):83-9.

27. Smolinska MJ, Page TH, Urbaniak AM, Mutch BE, Horwood NJ. Hck tyrosine kinase regulates TLR4-induced TNF and IL-6 production via AP-1. J Immunol. 2011;187(11):6043-51.

28. Bai Y, Zang X, Ma J, Xu G. Anti-Diabetic Effect of Portulaca oleracea L. Polysaccharideandits Mechanism in Diabetic Rats. Int J Mol Sci. 2016;17(8):1201.

29. Yang X, Yan Y, Li J, Tang Z, Sun J, Zhang H, et al. Protective effects of ethanol extract from Portulaca oleracea $\mathrm{L}$ on dextran sulphate sodium-induced mice ulcerative colitis involving antiinflammatory and antioxidant. Am J Transl Res. 2016;8(5):2138-48.

30. Ramadan BK, Schaalan MF, Tolba AM. Hypoglycemic and pancreatic protective effects of Portulaca oleracea extract in alloxan induced diabetic rats. BMC complement altern med. 2017 Jan 11;17(1):37. PubMed PMID: 28077129.

31. Couper KN, Blount DG, Riley EM. IL-10: the master regulator of immunity to infection. J Immunol. 2008;180(9):5771-7.

32. Sanjabi S, Zenewicz LA, Kamanaka M, Flavell RA. Antiinflammatory and pro-inflammatory roles of TGF- $\beta$, IL- 10 , and IL22 in immunity and autoimmunity. Curr Opin Pharmacol. 2009;9(4):447-53

33. Sabat R, Grütz G, Warszawska K, Kirsch S, Witte E, Wolk K, et al. Biology of interleukin-10. Cytokine growth F R. 2010;21(5):331-44.

34. Carter NA, Rosser EC, Mauri C. Interleukin-10 produced by B cells is crucial for the suppression of Th17/Th1 responses, induction of T regulatory type 1 cells and reduction of collagen-induced arthritis. Arthritis Res Ther. 2012;14(1):R32. 